$\xi=-1$

\title{
Characterization of Wavelet Decomposition Strain Signal Using the K-Mean Clustering Method
}

\author{
A. A. Rahim*, C. H. Chin, S. Abdullah, S. S. K. Singh, M. Z. Nuawi and F. H. A. Hassan \\ Centre for Integrated Design for Advanced Mechanical System (PRISMA) \\ Faculty of Engineering \& Built Environment, Universiti Kebangsaan Malaysia \\ 43600 UKM Bangi, Malaysia. \\ *Corresponding author Email: airee.afiq@siswa.ukm.edu.my
}

\begin{abstract}
This paper aims to study the characterisation of time-frequency domain to analyse the fatigue strain signal due to weaknesses in time domain and frequency domain approaches. The objectives were to determine the behaviour of strain signal, characterise the fatigue life of strain signal and validate the fatigue life in time-frequency domain. The strain signal was obtained using data acquisition devices and strain gauges on two types of road condition including highway and industrial area. The acquired signals were analysed with time domain, frequency domain and time-frequency domain approaches. In time-frequency domain, the signals were decomposed using 4th Daubechies discrete wavelet transform. To validate the effectiveness of time-frequency approach in characterising vibration fatigue signal, fatigue data was clustered by mapping of the data based on the spectrum energy, root-mean-square and fatigue life obtained. The clustering was performed by comparing the centroid values which both data had five clusters as the optimum data clustering with 0.836 average distance to centroid. From this, the relationship between fatigue life, root-mean-square and spectrum energy can be determined and thus a new fatigue life criterion was developed.
\end{abstract}

Keywords: Fatigue life; frequency domain; $k$-mean clustering; time-frequency domain; wavelet decomposition.

\section{Introduction}

In a ground vehicle, the suspension system absorbs the vibration and protects the vehicle body from damage during vertical movement of the wheels. Moreover, other components such as transmission and bracket also play important role by supporting suspension system to give a smooth and comfortable ride to the users [1]. Based on previous studies, the component performance was affected by the surface road experienced by the component when the vehicle moving and this phenomenon will lead to fatigue failure [2]. Thus, it is important to predict fatigue damage at early design stage as it helps in improving the durability of the components.

Failure analysis is able to detect fatigue damage in a component which subjected to repeated load cycles. Various approaches in time domain and frequency domain are available for fatigue analysis. Time domain approaches require input of time histories but these approaches need a large data amount to approximate the statistics behaviours [3]. Hence, frequency domain approaches become another alternative for fatigue analysis other than time domain approaches [4]. In frequency domain, power spectral density (PSD) gives the average square amplitude of every sinusoidal wave of each frequency in the signals. Frequency domain approaches are able to reveal the amplitude of signal frequency but these approaches do not give information of time in which the frequency is occurring [5]. In addition, most of automotive fatigue signals are non-stationary. Thus time-frequency domain analysis is more suitable to analyse fatigue data due to its ability to identify the high fatigue activities in time and frequency basis [6].
In this study, fatigue life of the coil spring were characterised in time-frequency domain in order to correlate the parameter used in all domain stated. The discrete wavelet transform is developed by using Daubechies method to extract the characteristics of the signals [5]. In addition, based on time-frequency analysis, the fatigue life of the component is validated by comparing with three different domains. Based on these, the characterization of fatigue vibration signal can be achieved in time-frequency domain.

\section{Methodology}

The strain loading histories of coil spring were collected as the car was driven on two different road surfaces including the highway and industrial area. Figure 1 shows the location of strain gauge attached on the coil spring. The signals were sampled at $500 \mathrm{~Hz}$ for 60,000 data points which gave a total signal length of 120 seconds. Subsequently, time domain, frequency domain and timefrequency domain approaches were adopted to analyse the signals. For the time domain analysis, the strain signals were analysed by observing the behaviour and fatigue life of the signals was determined using commercial software.

In time domain approach, fatigue life was predicted using strainlife approaches including the Coffin-Manson relationship, SmithWatson-Topper (SWT) and Morrow models. Strain-life approaches were used with Palmgren-Miner's linear cumulative damage rule as shown in Equation (1) [7].

$$
D_{N c}=\sum_{i=1}^{k} \frac{n_{i}}{N_{i}}
$$


where $D$ is the damage value, $N$ is total number of cycles and $n_{i}$ is the number of applied cycles.

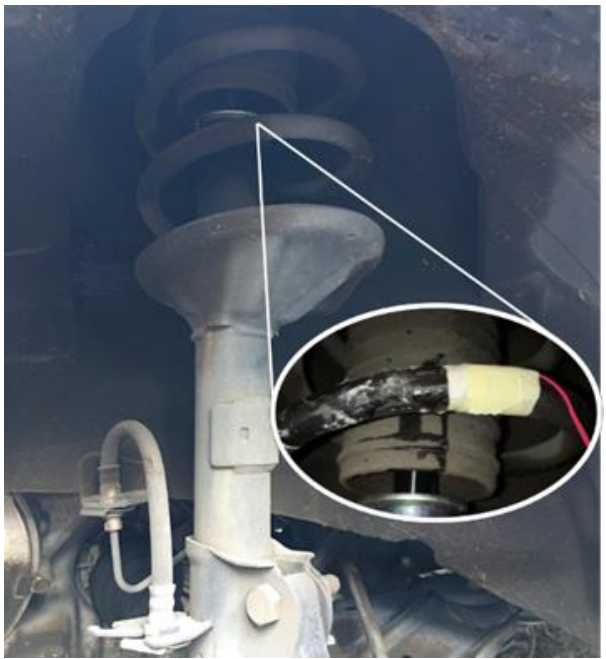

Fig. 1: Example of an image with acceptable resolution

The Coffin-Manson relationship, SWT and Morrow models are expressed in Equation (2), (3) and (4), respectively.

$\varepsilon_{a}=\frac{\sigma_{f}^{\prime}}{E}\left(2 N_{f}\right)^{b}+\varepsilon_{f}^{\prime}\left(2 N_{f}\right)^{c}$

$\sigma_{\max } \varepsilon=\frac{\left(\sigma_{f}^{\prime}\right)^{2}}{E}\left(2 N_{f}\right)^{2 b}+{\sigma^{\prime}}_{f}{\varepsilon^{\prime}}_{f}\left(2 N_{f}\right)^{b+c}$

$\varepsilon=\frac{\sigma_{f}^{\prime}-\sigma_{\text {mean }}}{E}\left(2 N_{f}\right)^{b}+\varepsilon_{f}^{\prime}\left(2 N_{f}\right)^{c}$

where $\varepsilon_{a}$ is the true strain amplitude, $\sigma$ is the fatigue strength coefficient, $b$ is the fatigue strength exponent, $\varepsilon_{f}^{\prime}$ is the fatigue ductility coefficient, $c$ is the fatigue ductility exponent, $E$ is the Young's modulus and $2 N_{f}$ is the number of cycle to failure. The fatigue life in Coffin-Manson relationship was calculated based on strain amplitude at zero mean stress. SWT and Morrow models considered the mean stress effect in the calculation of fatigue life. The correction of mean stress effect of Morrow is more realistic. The fatigue damage can be calculated with Equation (5):

$D=\frac{1}{N_{f}}$

where $D$ is fatigue damage and $N_{f}$ is the number of cycle to failure.

In time series, the signal can be analysed by observing the changes of the signal from time to time. Statistical analysis is one of the methods to analyse the signal and obtain statistical parameters such as mean, root-mean-square (RMS) and kurtosis. The equations for these statistical parameters are expressed as following:

$$
\begin{aligned}
& \text { Mean, } \bar{x}=\frac{1}{n} \sum_{j=1}^{N} F \\
& R M S=\left\{\frac{1}{N} \sum_{j=1}^{N} x_{j}^{2}\right\}^{\frac{1}{2}} \\
& \text { Kurtosis }=\frac{1}{N(R M S)^{4}} \sum_{j=1}^{N}\left(x_{j}-\bar{x}\right)^{4}
\end{aligned}
$$

Where $F$ is the signal with the number of data, $n$.

In order to perform frequency domain analysis, the PSD of the signals were obtained to get the energy distribution in the signals. The process included the conversion of time domain signal into the frequency domain. For most fatigue studies, the frequency domain analysis is done using PSD. It is a spectrum analysis that considered the energy of a signal in the frequency domain. PSD can be expressed as followed:

$$
P S D=\frac{1}{2 \pi} \sum_{n=-\infty}^{\infty} F(n) e^{-i \omega t}
$$

where $F$ is the signal with the number of data $n, t$ is the time and $\omega$ is the angular frequency.

Decomposition of the signals was performed using Daubechies $4^{\text {th }}$ order with 12 levels of decompositions which are optimal levels to remove most of its noise. Wavelet decomposition calculates the group index known as wavelet coefficient [8]. The coefficients obtained from the signal regression generated at different frequency scales in a wavelet. The signal generated establish the correlation between the wavelet and a section of the signal being analysed. The Daubechies wavelet transform of class $D-2 N$ is a function $\varphi=N^{\varphi} \in L^{2}(\Re)$ defined as followed:

$$
\varphi(x)=\sqrt{2} \sum_{k=0}^{2 N-}(-1)^{k} h_{2 N-1-k} \varphi(2 x-k)
$$

where $h_{0}, \ldots, h_{2 N-1} \in \mathfrak{R}$ and $N$ is the order.

For each level, the spectrum energy, fatigue life and RMS of the signal were obtained to analyse the characteristics of the signals. Then, these parameters were used as the input for the data clustering process using k-mean algorithm.

\section{Results and Discussion}

Figure 2 and 3 shows the strain signal obtained from the coil spring for two types of road which were highway and industrial area, respectively. Statistical parameters are able to classify the strain signals of highway and industrial area. Table 1 shows the statistical result for both road conditions. Based on the observation and statistical result, the signals can be classed as tension strain signal since the signals had positive mean value. Thus, both signals can be defined as tension since they had positive mean value. Other than that, it was found that the both signals were nonstationary as their kurtosis values were more than three. Normally, kurtosis of a Gaussian distribution is 3.0. The signal distribution contained high peak activities that contribute to damage if its kurtosis is higher than 3.0. In industrial area signal, there was more transient event associated with large and sudden amplitude changes compared to the highway road signal. This is attributed to the bumpy surface and potholes in industrial area.

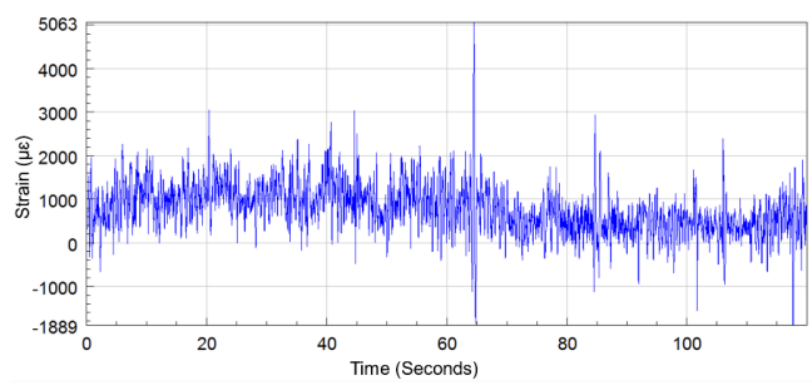

Fig. 2: Strain signal obtained from highway 


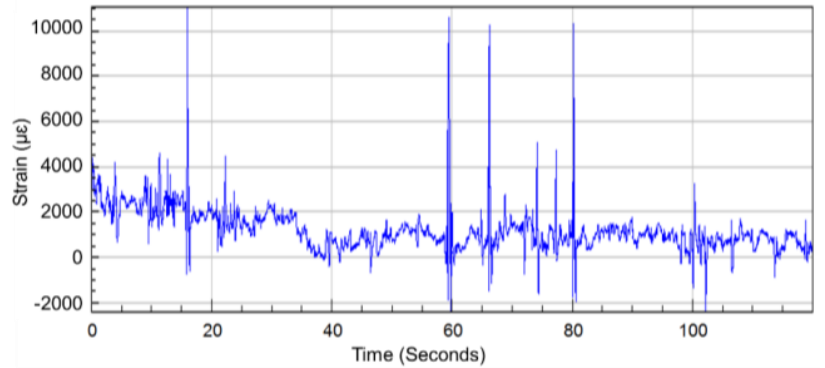

Fig. 3: Strain signal obtained from industrial area

Table-1. Statistical analysis for highway road.

\begin{tabular}{|c|c|c|}
\hline Statistic parameter & Highway road & Industrial road \\
\hline Mean, $\mu \varepsilon$ & 3847.69 & 1219.18 \\
\hline Kurtosis & 5.9719 & 27.6382 \\
\hline RMS, $\mu \varepsilon$ & 4704.72 & 1569.88 \\
\hline
\end{tabular}

Fatigue life was predicted based on the three strain-life approaches. The fatigue life was measured in number of cycle until failure. Table 2 shows the fatigue life for each signals obtained by using commercial software. The strain-life model was chosen based on the mean value of signals. This model relates deformation occurring in the immediate vicinity of a stress concentration to the remote or local pseudo-elastic stresses and strains using the constitutive response determined from fatigue failure [9]. Therefore, Smith-Watson-Topper (SWT) model was used to determine both fatigue life. Morrow model is used if the signal has a negative mean value while Coffin-Manson is suitable for zero mean value [10]. Therefore, highway road give higher life cycle with $5.26 \times 10^{5}$ cycles compared to industrial road with $2.88 \times 10^{5}$ cycles.

Table-2. Fatigue life for each data of highway road.

\begin{tabular}{|c|c|c|c|}
\hline \multirow{2}{*}{ Road condition } & \multicolumn{3}{|c|}{ Fatigue life (Number of cycles) } \\
\cline { 2 - 4 } & $\begin{array}{c}\text { Coffin- } \\
\text { Manson }\end{array}$ & SWT & Morrow \\
\hline Highway & $5.85 \times 10^{5}$ & $5.26 \times 10^{5}$ & $5.51 \times 10^{5}$ \\
\hline Industrial area & $4.07 \times 10^{5}$ & $2.88 \times 10^{5}$ & $3.29 \times 10^{5}$ \\
\hline
\end{tabular}

By using power spectral density (PSD) analysis, frequency content of the signals can be obtained by using Fourier transform algorithm [4]. Figure 4 and 5 depict the PSD of highway and industrial area signals, respectively. Based on the figures, high amplitudes were found at low frequency in highway signal. Therefore, the characteristics of the signal is influenced by the high amplitude that occurred at low frequency range. From the PSD, spectrum energy was calculated from area under the graph and tabulated in Table 3 [11]. The spectrum energy represents the energy content of a signal. The energy contents in the signal were affected by the road surface features such as bumps and potholes on the road which contribute to fatigue damage in the coil spring.

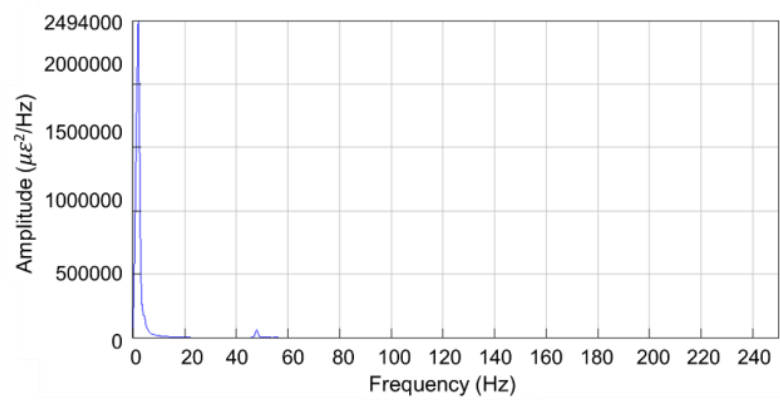

Fig. 4: PSD of highway road signal

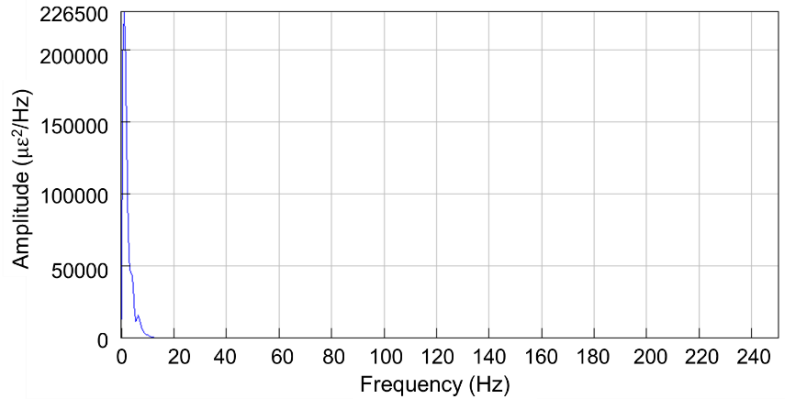

Fig. 5: PSD of industrial road signal

Table-3. Statistical analysis for highway road.

\begin{tabular}{|l|c|}
\hline Road condition & Energy $\left(\mu \varepsilon / \mathrm{Hz}^{2}\right)$ \\
\hline Highway & $4.90 \times 10^{4}$ \\
\hline Industrial area & $5.52 \times 10^{5}$ \\
\hline
\end{tabular}

The strain signals were decomposed to 12 levels using 4th order Daubechies wavelet transform based on the number of data for the particular signal. The statistical analysis of the decomposed signals was remained the same as the original signal in order to classify the signal to its own frequency [6]. Figure 6 and 7 shows the decomposed signals of highway and industrial area up to 12 level of decomposition, respectively. The fatigue life of each level was calculated and tabulated in Table 4. From Table 4, it was found that the fatigue life increases as the level of decomposition increases compared to the original fatigue life for both signals. This was because the fatigue damage in higher level was minimal, thus resulted in longer fatigue life.

All the data were combined and clustered using k-means method by considering the spectrum energy of PSD, RMS and fatigue life for each levels of decomposition. Every parameters was normalized to ensure all the values were in similar range. Figures 8-11 show the fatigue data clustering with several clusters. As the total of cluster increases, the distance of the cluster from the centroid decreases [12]. The distance shows the significant relationship between data and centroid in each group which also known as the objective function. Table 5 shows the average distance of the data from the centroid. From the analysis, number of clusters five gave the lowest objective function value.

Table 4: Fatigue life of original and decomposed signals at different decomposition level

\begin{tabular}{|c|c|c|}
\hline \multirow{2}{*}{ Level of decomposition } & \multicolumn{2}{|c|}{ Fatigue life (cycle) } \\
\cline { 2 - 3 } & Highway & Industrial area \\
\hline Original & $5.511 \times 10^{5}$ & $3.298 \times 10^{5}$ \\
\hline Level 1 & $3.393 \times 10^{5}$ & $3.738 \times 10^{5}$ \\
\hline Level 2 & $3.259 \times 10^{5}$ & $3.341 \times 10^{5}$ \\
\hline Level 3 & $1.377 \times 10^{5}$ & $3.905 \times 10^{5}$ \\
\hline Level 4 & $9.056 \times 10^{4}$ & $5.463 \times 10^{5}$ \\
\hline Level 5 & $6.770 \times 10^{4}$ & $4.718 \times 10^{5}$ \\
\hline Level 6 & $6.486 \times 10^{4}$ & $5.941 \times 10^{5}$ \\
\hline Level 7 & $1.097 \times 10^{5}$ & $1.549 \times 10^{6}$ \\
\hline Level 8 & $2.157 \times 10^{6}$ & $7.677 \times 10^{7}$ \\
\hline Level 9 & $2.699 \times 10^{6}$ & $1.615 \times 10^{10}$ \\
\hline Level 10 & $9.645 \times 10^{6}$ & $2.561 \times 10^{10}$ \\
\hline Level 11 & $2.228 \times 10^{7}$ & $1.657 \times 10^{11}$ \\
\hline Level 12 & $1.514 \times 10^{8}$ & $5.669 \times 10^{11}$ \\
\hline
\end{tabular}

Table 5: Average distance of data to centroid

\begin{tabular}{|c|c|}
\hline Number of cluster & Average distance to centroid \\
\hline 2 & 4.261 \\
\hline 3 & 2.619 \\
\hline 4 & 1.587 \\
\hline 5 & 0.836 \\
\hline
\end{tabular}

The optimum objective function value for the centroid of the cluster is 0.836 . This value shows the distribution of the data clustered were focus on the centroid as shown in Figure 11. Therefore, the decomposed signals can be easily categorized in three groups which are; group 1 is high spectrum energy with high RMS value, group 2 is moderate spectrum energy with moderate RMS value, and group 3 is low spectrum energy with low RMS value based on 
the patterns shows on each figures. Thus, the high amplitude signal contained high energy is the main factor in contribution to fatigue failure to occur. The data that were scattered far away from the groups can be neglected as its do not have significant values because the characteristics of the data were differ from the clustered data.

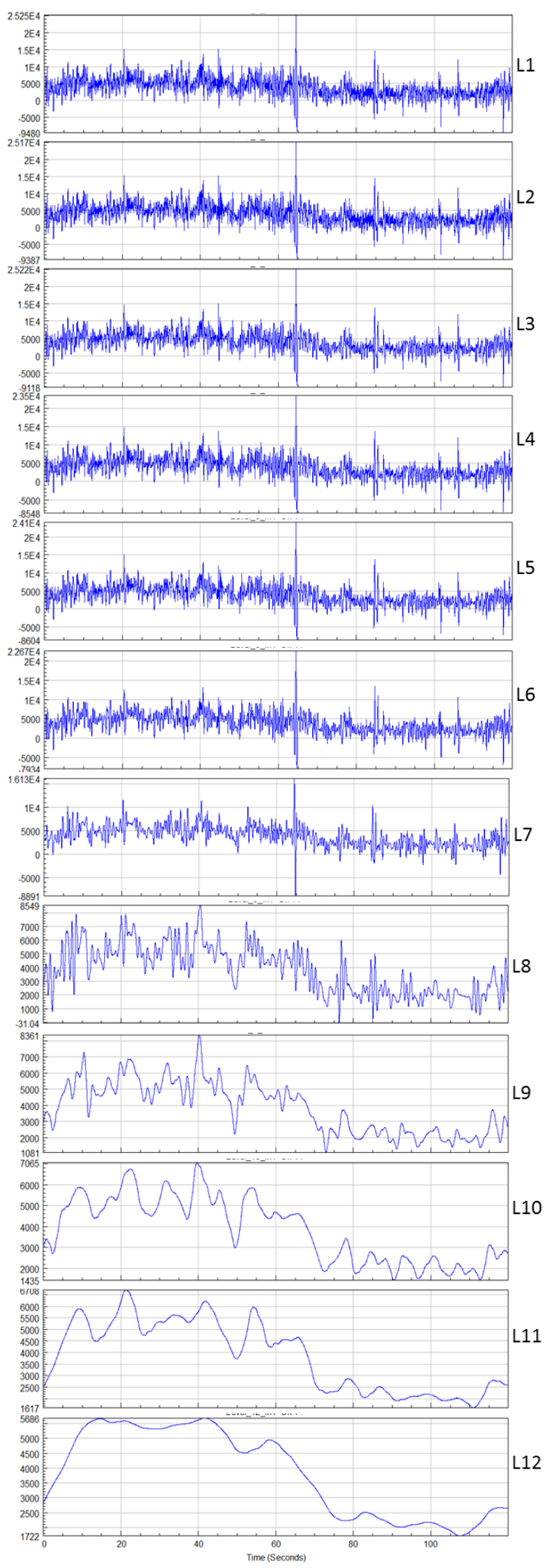

Fig. 6: Decomposition of highway signal using DWT

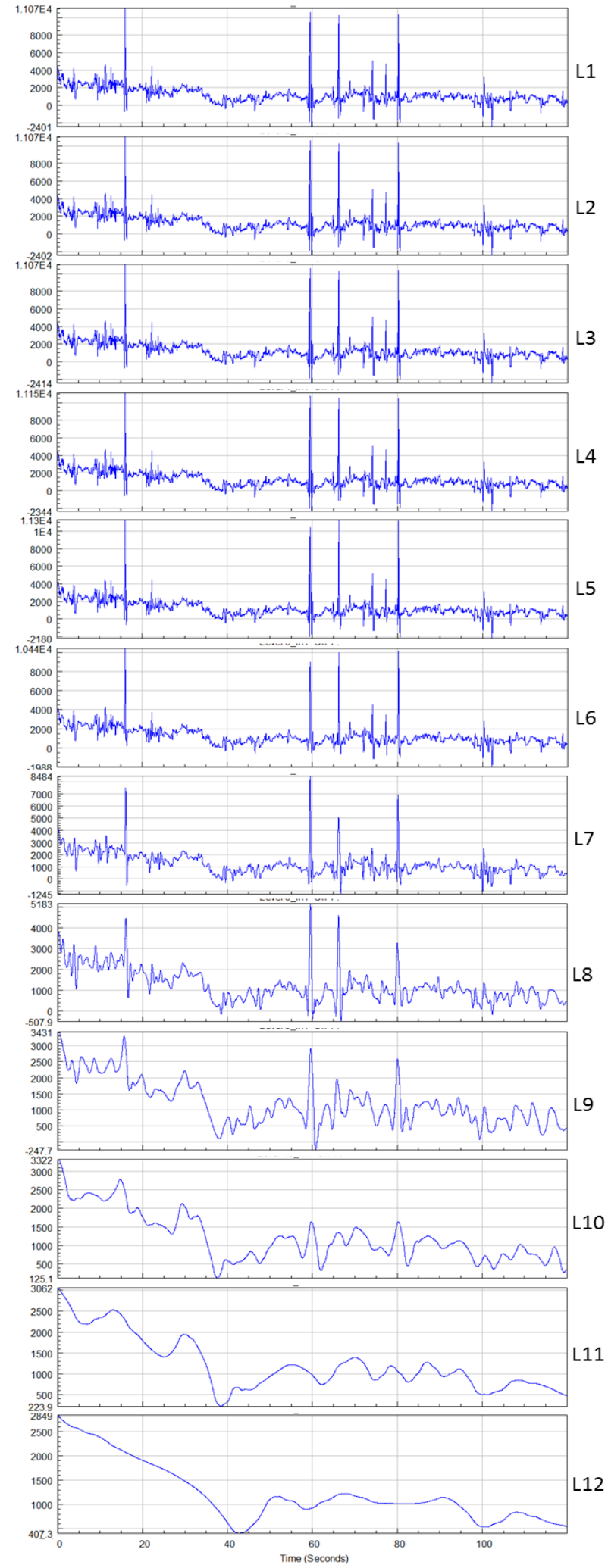

Fig. 7: Decomposition of industrial area signal using DWT

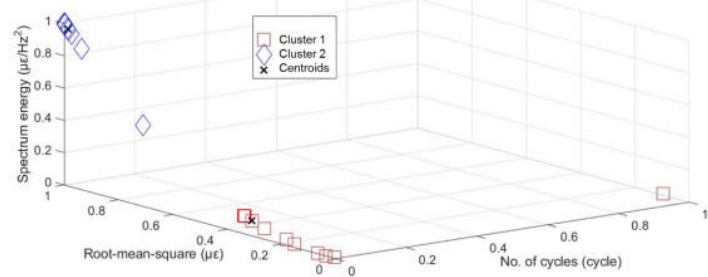

Fig. 8: Two clusters for normalised number of cycles, normalised RMS and normalised energy using k-means clustering approach in $3 \mathrm{D}$ 


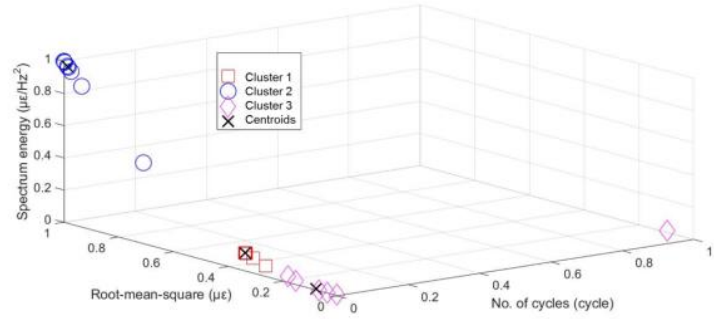

Fig. 9: Three clusters for normalised number of cycles, normalised RMS and normalised energy using k-means clustering approach in 3D

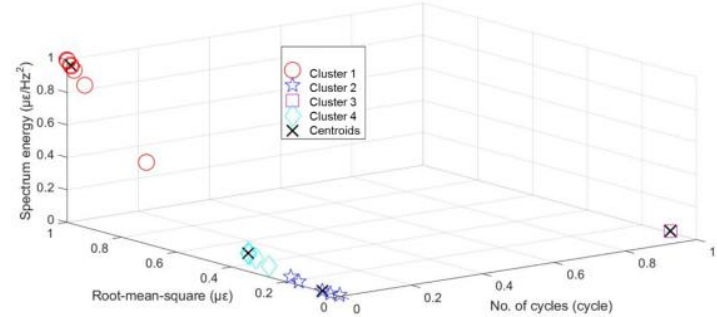

Fig. 10: Four clusters for normalised number of cycles, normalised RMS and normalised energy using k-means clustering approach in $3 \mathrm{D}$

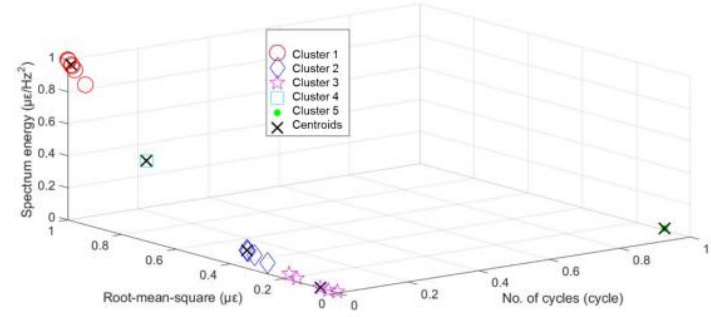

Fig. 11: Five clusters for normalised number of cycles, normalised RMS and normalised energy using k-means clustering approach in $3 \mathrm{D}$

\section{Conclusion}

In this study, two signals representing the highway and industrial area road were obtained. The road surfaces play a significant role in determine to the characteristics of the signals. Based on the data collected, industrial area signal has shorter fatigue life of $2.88 \mathrm{x}$ $10^{5}$ compared to highway signal $\left(5.26 \times 10^{5}\right)$. From the time histories, the high peak amplitude with low background signal contributes to high fatigue. In frequency domain approach, PSD was utilized to obtain the spectrum energy in the signals to identify the signal that has high vibration energy. From the analysis, the characteristics of the fatigue life in the signal can be identified when the high amplitude occurs at low frequency. In addition, the signal was analysed by decomposing the signals using Daubechies wavelet transform. Spectrum energy, RMS and fatigue life the signal at each level of decomposition were used as parameter in fatigue data clustering. The optimum group of clusters were found to be five for both signals and the comparison shows similar characteristics. Therefore, it can be concluded that the Daubechies method is suitable to access the characteristic of fatigue life.

\section{References}

[1] Patil R, Ingawale S, Rupnar R \& Tupake S (2015), Design and analysis of independent suspension system using FEA. International Journal of Engineering Research \& Technology 4(4), 769-775.

[2] Al-Asady NA (2009), Comparison between experimental road data and finite element analysis data for the automotive lower suspension arm. European Journal of Scientific Research 29(4), 557- 571.

[3] Lei Y, He Z \& Zi Y (2009), Application of the EEMD method to rotor fault diagnosis of rotating machinery. Mechanical Systems and Signal Processing 23(4), 1327-1338.

[4] Bendjama H, Bouhouche S \& Boucherit MS (2012), Application of wavelet transform for fault diagnosis in rotating machinery. International Journal of Machine Learning and Computing 2(1), 82-87.
[5] Putra TE, Abdullah S, Schramm D, Nuawi MZ \& Bruckmann T (2017), Reducing cyclic testing time for components of automotive suspension system utilising the wavelet transform and the fuzzy c means. Mechanical Systems and Signal Processing 90, 1-14.

[6] Oh C (2001), Application of wavelet transform in fatigue history editing. International Journal of Fatigue 23, 241-250.

[7] Apetre N, Arcari A, Dowling N, Iyyer N \& Phan N (2015), Probabilistic model of mean stress effects in strain-life fatigue. Procedia Engineering 114, 538-545.

[8] Medina-Daza RJ, Vera-Parra NE \& Upegui E (2017), Wavelet daubechies (db4) transform assessment for worldview-2 images fusion. Journal of Computers 12(4), 301-308.

[9] Santagati S, Bolognini D \& Nascimbene R (2012), Strain life analysis at low-cycle fatigue on concentrically braced steel structures with rhs shape braces. Journal of Earthquake Engineering 16, 107 137.

[10] Yahya MM, Mallik N, \& Chakrabarty I (2015), Life prediction of low cycle fatigue behavior in rotating cantilever beam of Al- Alloy AA 6063-T6 at room temperature. International Journal of Emerging Technology and Advanced Engineering 5(11), 95-103.

[11] Liberatore S \& Carman GP (2004), Power spectral density analysis for damage identification and location. Journal of Sound and Vibration 274, 761-776.

[12] Napoleon D \& Pavalakodi V (2011), A new method for dimensionality reduction using k-means clustering algorithm for high dimensional data set. International Journal of Computer Applications 13(7), $0975-0987$. 